monosyllable unwittingly provided a clue to the nature of his genius.

Dirac's ideas are invariably very simple, simpler indeed than usual preconceptions of distance, position, time and so on, and followed up with complete literalness and disregard of commonsense. He deduced the existence of antimatter by a very long argument which began with a deduction that the wave equation must be first order in the distance variables. At that point, where most physicists would have written "which is absurd", Dirac wrote "therefore".

Dirac's work of forty years ago showed that all particles of a certain degree of simplicity have no internal degrees of freedom other than a spin, which must be half integral provided that the position of the particles is, in a certain sense, observable: this is true of electrons.

Dirac, in his new articles, allows more internal degrees of freedom characteristic of two harmonic oscillators and proceeds to write down the simplest conceivable wave equation consistent with that assumption. This equation bears a strong formal resemblance to the Dirac equation for the electron. It is a tribute to the methods discovered forty years ago that exactly the same methods elucidate quite different properties deduced from the new hypothesis and show that the resemblance was entirely formal. The new particles have positive mass, integral spin, zero orbital angular momentum and spherical symmetry.

It is evident that the fertility of the mathematical methods discovered by Dirac when a young man is not yet exhausted. But, recalling his remarks to the journalist, further explanation will not be attempted here.

\section{AGGRESSION \\ Keeping Up the Rivalry}

from our Animal Behaviour Correspondent

THERE has been much controversy recently about the causation of aggression in animals. Konrad Lorenz, in his book On Aggression, claims that animals have an aggressive "drive" which builds up and is discharged through acts of aggression or their substitutes. His view of aggression is of a force within the animal, as inevitable and basic as hunger, and something which, if not allowed expression, will build up to dangerous levels. Other authors, such as the American J. P. Scott, see the aggression of an animal as being elicited by external stimuli and unlikely to occur unless these stimuli are provided.

W. Heiligenberg and U. Kramer ( $J$. Comp. Physiol., 77, 332; 1972) have performed some experiments which go strongly against Lorenz's views of a spontaneous build up of aggression and illustrate for the cichlid fish Haplochromis burtoni the great importance of the right external stimuli in keeping the aggressive tendency high. They used the rate at which male fish would attack small test fish as a measure of how aggressive the males were. If the males were isolated from other adults for a few weeks, their aggressiveness fell to a very low level, which is, of course, the very opposite of what would be expected if their aggressiveness were, as Lorenz claims, spontaneously "building up". The external stimulus of another adult fish seems to be needed to keep aggression high.

Functionally, this behaviour would be favoured by natural selection. When a male holding a territory is approached by a rival, his readiness to attack will be increased and this will in turn raise the probability of his winning the encounter. The aggressiveness will thus be adjusted to the number of encounters with other males in which a male has to participate in order to keep the territory free of rivals.

\section{ACETYLCHOLINE}

\section{Dual Action Synapse}

from our Neurochemistry Correspondent

THE use of comparatively simple preparations from the nervous system of invertebrates has many advantages for the study of basic mechanisms of synaptic transmission, chiefly because of the relative precision with which connexions can be described. The ganglia of Aplysia have proved an outstanding example, although from the richness of the responses of preparations to applied chemicals, they may have seemed to be something of a pharmacologist's Pandora's box. The use of such preparations, however, is vindicated in an article describing a novel type of chemical synapse discovered in the buccal ganglion of Aplysia, where the same transmitter causes concurrent excitation and inhibition (D. Gardner and E. R. Kandel, Science, 176, 675; 1972).

In the ganglia of A plysia single neurones innervate a large population of follower cells. Kandel and his col-

\title{
Differential Recognition Immune Responses
}

IT is common among the various groups which study tumour immunology to suppose that the putative response of a tumour-bearing organism to its tumour involves two components, humoral and cellular. It is further envisaged that the cellular component consists of cytotoxic lymphocytes of which the activity may be either impeded or potentiated by antibody. This hypothesis, comfortable in the sense that it can be used to fit all occasions, also usefully points to the necessity for measurement of both components of the immune process.

It is clear that, if the hypothesis bears any relation to the truth, it is desirable to interfere with the response to the tumour, not by some blanket type of immunosuppressive or immunopotentiating regimen but by effecting separate regulation of the two components of the response. For example, if antibodies are blocking what would otherwise be an effective cytotoxic cell population, the antibody response must be reduced without concomitant reduction of activity by the cytotoxic cells. In a somewhat indirect way the report by Thompson et al. in Nature New Biology next Wednesday (July 5) impinges on this problem.

The humoral immune responses of guinea-pigs to lysozyme and its reduced and S-carboxymethylated derivative (CM-lysozyme) consist of a non-crossreactive array of antibody molecules. Thompson et al., however, show that in other ways the immune response to lysozyme and the partly denatured CMlysozyme is cross-reactive. Spleen cells from immunized animals responding in vitro to either the homologous or heterologous antigen, for example, give exactly the same response, as measured by incorporation of tritiated thymidine. Delayed skin reactions of differentially immunized animals were almost exactly alike, whichever of the two species of antigen was used to elicit the recall response.

The production of a factor which inhibits the migration of peritoneal exudate cells could be successfully elicited from sensitized cells whichever of the antigens was added to the reacting cells. Mice which had been rendered tolerant to lysozyme were found to be cross-tolerant to CM-lysozyme.

The inference from these findings is that although the antigenic recognition system which is involved in humoral antibody production failed to find any recognition sites in common between lysozyme and CM-lysozyme, nevertheless the recognition system(s) which was or were involved in the other tests did find common sites.

Thompson et al. point out that their findings indicate a fundamental difference in the mode of recogition of antigens which induce humoral and cellular immunity. They specifically suggest that this may be of prime importance in situations in which it is wished to augment a cellular response by use of a partly denatured immunogen, without affecting the humoral response which, on their evidence, will not recognize any determinants in common in the native and denatured material. 